\title{
How complete are our clerkings? A project aimed at improving the quality of medical records by using a standardised proforma.
}

\author{
Jij Chow, Camille Yvon, Tim Stanger \\ Medway NHS Trust
}

\begin{abstract}
Clerking a patient is the first and most important contact that any medical team has with the patient. It provides information that may not be available later in the admission if recorded improperly, such as referral letters, collateral history, dosette boxes or prescription sheets. The quality of data recorded from this encounter was examined at a busy district general hospital surgical department. Initial measurement demonstrated that less than half of certain key parameters like dosages of medication were being recorded by clerking doctors. A clerking proforma was therefore designed to combat this problem. Over three audit cycles $(n=170,150,174)$ the proforma showed statistically significant improvements in the proportion of data that was captured at initial contact with the patient. We conclude that the introduction of proformas for clerking significantly improves the collection of data that impacts patient care during their hospital stay.
\end{abstract}

\section{Problem}

At the Medway Maritime Hospital, a district general hospital in Kent, UK, clerkings in the surgical department have traditionally been done on plain paper. An initial audit by the authors demonstrated that clerking standards were deficient. Key features like allergies were not being noted. For more detailed explanations of the criteria, please see the section 'Baseline Measurement'.

\section{Background}

Clerking is usually the first point of information gathering by a clinician about a patient's condition before hospital admission. It is the exchange that results in a provisional diagnosis and management plan. First admission to hospital is usually the easiest time to take a complete history, as patients often come in with relatives, prescription sheets, and letters from GPs. Therefore the completeness of clerkings is very important - without full information there may be delayed or even poor decision making. For context, an example patient pathway is detailed in Figure 1.

\section{Baseline Measurement}

The initial audit assessed clerking standards to the following standards:

1. All patients should have a recorded allergy status

2. All patients should have a list of medications

3. All patients should have doses recorded by appropriate medications

4. All patients should have a documented social history specifying if they are independent and mobile. An explanation or clarification should be given if this is not the case.

5. All patients should have a documented plan for oral intake, ie, nil by mouth, free fluids, eat and drink, etc.
Standards 1-4 were tested on the clerking doctor's entry, and standard 5 was tested on the first senior review (either a registrar or a consultant). In the case of a senior doctor being the clerking doctor, the first entry was considered as both. Data were collected only for surgical, vascular, and urology specialties. Elective surgery patients were admitted independent of the surgical assessment unit (SAU) and were hence not included in this audit.

In January 2013 data were collected four random days a week, morning and night, to sample the clerkings of a variety of both day and night teams. An auditor would go through all the new surgical patients to come through SAU and check them against the above standards. The data were inputted to a computer spreadsheet for analysis.

In all, data were collected on the clerkings of more than 30 doctors. To avoid duplication, the date, patient initials, clerking specialty, and grade of doctor were also recorded and double entries were eliminated using a spreadsheet at the end of collection. Note that if the patient was on no regular medications, the doses were considered to have been recorded. The initial results are displayed alongside the second cycle results in Table 1 and Figure 2.

\section{Design}

During the first cycle of our audit, it became clear that the standards of surgical clerking at Medway hospital fell short of acceptable. We felt that the practice of using plain paper rather than a formal clerking proforma could be partly responsible for this.

A surgical clerking proforma was created in collaboration with $\mathrm{Mr}$ Liam Poynter and Mr Chris Butler (two of the senior surgeons at the Trust) and then trialled in the SAU. It was structured via a series of clear headings, following criteria set by the Royal College of Surgeons. It was designed to act as a prompt for key parts of the history and examination and to facilitate information recovery further down the patient's care pathway. After the proforma was designed, 
it was printed and sent to the SAU. Funding for this was provided by the ward.

\section{Strategy}

PDSA cycle 1: The initial results were analysed, showing deficiency in information recording. Clerking proformas from different specialties and different sites were examined, as well as the Royal College of Surgeons 1994 guidelines on medical records. With L Poynter and C Butler, a proforma was created, printed, and distributed to the SAU.

PDSA cycle 2: After printing the initial copies, staff were consulted in a meeting to evaluate their views and gain suggestions. Using these recommendations and following discussion with Poynter and Butler, the proforma was edited and a second batch was printed for use.

PDSA cycle 3: The second audit cycle demonstrated statistically significant improvements in nearly all fields. The allergy field did not improve due to a printing error in which it was 'greyed out'. This was corrected and a new batch printed. The audit was presented at two large Trust meetings, where it was possible to gain more constructive feedback.

PDSA cycle 4: The third audit cycle was performed as a postmeasurement. The allergy section had improved by a statistically significant amount. Other standards had stayed statistically the same. Discussion with auditors from other trusts revealed education sessions to be of use in improving the figures. This is the planned action in the coming months.

\section{Results}

One hundred and fifty-three patient files were recorded over the second cycle, of which three were excluded due to duplication, leaving 150 samples.

The second cycle after the introduction of the proforma showed four statistically significant improvements: medication recording improved by $16 \%$, dosage by $43 \%$, social history by $54 \%$, and nutritional plan by $28 \%$ ( Tables 1 and 2, Figure 2,). Allergy recording got worse by a non-statistically significant difference $(p=$ $0.282)$.

Contrary to cycle 1 , house officers outperformed senior house officers in use of the clerking proforma in cycle 2 (cycle 1 data: house officers $66.4 \%$, senior house officers $70.0 \%$ ). Registrars once again proved worst at filling out the information and obtained poorer results than the previous cycle where they scored $45.4 \%$ (Table 3).

Cycle 3 was performed 1 year after introduction of the proforma $(n=174)$. The only statistically significant difference was the improvement in allergy recording (Figure 2, Table 4 and 5). This was very encouraging. Also the other standards had not changed significantly, indicating that performance had been maintained over a year of use.

Qualitatively it was noticed that information was far easier to find, because the proforma was well marked out and provided a single document in which all the relevant information could be obtained. In addition, it pushed the clerking doctor to follow a systematic and logical approach, hence speeding up clerking.

See supplementary file: ds2984.docx - "Figures and tables v1"

\section{Lessons and Limitations}

Our audit was limited, as not all data were collated from the target days. The main losses of data were surgical patients clerked in accident and emergency (A\&E), who were not admitted via the SAU, and patients who were discharged quickly after clerking and senior review. These notes were often unavailable to us on the unit, having been dispatched for filing. Nonetheless, this happened at random and missing data were unlikely to bias the result.

The ambitious target of obtaining full information on the clerking items tested was not reached. However, significant improvements were made by the introduction of the proforma. Most startlingly the doses of medications and the inclusion of a social history improved by greater than 20 percentage points apiece.

Allergy recording did not improve in the second cycle. We had expected a statistically significant increase, similar to the other recorded items. Reassuringly, the drop in recording was not statistically significant. Despite its size, the greying out of the allergy section may have decreased its visual impact on the page rather than highlighting it in a predominantly paper-white setting. It was also physically separate from the medications box, which may have reduced its value in prompting the questioning of the patient. The proforma was subsequently modified and showed encouraging results in the following cycles.

Another observation we made in the filling of the forms was that although nil-by-mouth decisions were better documented in the later cycles, the check boxes provided were often not used. Once again, the separation from the senior review box probably contributed to the lack of use.

We posted a sheet in the SAU doctors' office that invited staff to write suggestions for a new and improved version of the proforma. The multidisciplinary discussion group included doctors of various grades, nurses, and clinical support staff who all refer to the proforma.

Further discussion with auditors from other trusts has added the suggestion that educational sessions on how to use the proformas could be of benefit in improving compliance with the standards. This will be the future action for improving data collection at point of entry.

\section{Conclusion}




\section{BMJ Quality Improvement Reports}

Overall, the surgical admissions proforma significantly improved the quality of clerkings at Medway's assessment unit. Potential benefits include fewer medication errors, time saved in clerking, greater detail elicited from the patient encounter, enhanced interspecialty communication, and patient care. There are opportunities for further study in proving that clerking proformas actually influence endpoints such as medication errors, delays in surgery, and diagnosis. One of the authors is currently repeating the project at the Queen Elizabeth Hospital in London to ascertain if similar results can be achieved using a shorter and more succinct proforma, ideally reducing clerking times and improving ease of information finding.

\section{References}

1. Carpenter I, Ram MB. Admission record content structure standards for hospital practice: consultation questionnaire report, 2008. Health Informatics Unit, Royal College of Physicians of London.

2. RCSEng. Guidelines for Clinicians on Medical Records and Notes. London: Royal College of Surgeons, 1994.

\section{Declaration of interests}

Nothing to declare

\section{Acknowledgements}

Our fourth author, Dr Katy Simpson, who does not have a BMJ subscription.

Mr Liam Poynter, the leader of another audit and the major contributor to the proforma launched by the two projects.

With special thanks to the Surgical Assessment Unit ward clerks, Sr Deborah Fairweather (Head of SAU) and all the doctors and patients who talked, thought and wrote to create our data. 